\title{
On threefolds of linear genus unity.
}

\author{
Memoria di Leonard Roth (a Londra).
}

Summary. - This paper is devoted to a survey of the algebraic threefolds of absolute linear genus unity; they are divided into four main classes, and examples of each class are given.

It is a remarkable fact that, with the exception of the rational, and most of the scrollar, surfaces, nearly all the algebraic surfaces, regular or irregular, which have so far been studied, belong to one vast family. Those members of the family which possess an effective canonical or pluricanonical system are characterized by the property that their absolute linear genus is equal to unity; and, by an extension of the latter concept, it is possible to include even the elliptic scrollar surfaces in the scheme.

These results suggest that a useful beginning to the classification of algebraic threefolds might be made by examining those types for which the linear genus, suitably defined, is equal to unity. The present paper is devoted to a general survey of the problem; a detailed study of the various subclasses which arise has been left for subsequent investigation, and particular exampls have been mentioned only to illustrate specific points in the theory.

1. The surfaces for which $p^{(1)}=1$. - We begin by recalling the main facts (ENRIQUES, 4) concerning the classification of the surfaces of linear genus unity which will be required later.

Let $F$ be a surface which possesses an effective canonical or pluricanonical system of some index (possibly of order zero); then it is known that $F$ is birationally equivalent to some surface $G$ which is free from exceptional curves and for which it is therefore possible to define a pure canonical or pluricanonical system, as the case may be, and hence an effective linear genus, the absolute linear genus of $F$, which we denote by $p^{(1)}$. If, however, $F$ has no effective pluricanonical systems, in which case it is scrollar of genus $p \geq 0$, it has been shown by CASTELNuovo (2) that one may still define a character $p^{(1)}$ for the class of surfaces birationally equivalent to $F$, which is analogous to the former invariant; in fact, for $p=0, p^{(1)}=10$, while for $p>0, p^{(1)}=1-8(p-1)$.

With these conventions as to the meaning of the symbol, ENRIQUES has found that the surfaces for which $p^{(1)}=1$ belong to one of the following main classes: 
A. The first class consists of the elliptic scrollar surfaces, which are characterized by their arithmetic genus $p_{a}=-1$, and their 12-genus $p_{42}=0$.

The remaining surfaces of the family all possess canonical or pluricanonical systems of some index. In the first place we have

B. Surfaces for which $p_{12}=1, p_{a} \geq-1$.

These possess a canonical or pluricanonical curve of order zero. If $n, \pi$ denote the grade and genus of any curve system, without base points, on a model of this class which is free from exceptional curves, we have $n=2 \pi-2$; and the freedom of the complete system is $\pi, \pi-1$ or $\pi-2$, according as $p_{a}$ is equal to 1,0 or -1 .

A detailed examination shows that these surfaces fall into one or other of three sub-classes:

(1) Regular surfaces with genera and plurigenera unity; the general types all depend on 19 moduli and have base number unity, and are classified by means of the genus $\pi$ of the curve which forms the base. The prime sections are normal canonical curves.

(2) Surfaces whose prime sections are canonical but not normal; these are irregular hyperelliptic, with $p_{g}=1, p_{a}=-1$. The general types have base number unity.

(3) Surfaces whose prime sections are images of linear series which arise from division by an integer $s$ of the $s$-canonical series $(s=2,3,4,6)$.

These are either regular, in which case $p_{g}=p_{a}=0, p_{2}=1$, or elliptic, with $p_{g}=0, p_{a}=-1$; and they all contain pencils of elliptic curves. For this reason they are linked with the next class.

C. Surfaces for which $p_{12}>1$.

These contain pencils (rational or irrational) of elliptic curves, of which the canonical or pluricanonical systems are compounded. They include in particular the surfaces which possess a single canonical curve of genus 1 . The surfaces depend for their classification upon the following characters:

(i) The arithmetic genus $p_{a}$.

(ii) The determinant $d$, i. e. the set of points of minimum order which is rationally determinable on the generic curve of the pencil in question.

(iii) The multiple elliptic curves of the pencil; these fix the values of the plurigenera.

(iv) The genus of the pencil; when $p_{a} \geq 0$, this is equal to the irregularity $p_{g}-p_{a}$, but is equal to 1 in the case of a (special) hyperelliptic surface which contains elliptic curves; such a surface contains two pencils, both elliptic, of these curves. An elliptic surface for which $p_{g}=0, p_{a}=-1$, contains in general one pencil (linear) of elliptic curves; but type $B(3)$ contains in addition an elliptic pencil.

The classification of type $B$ is fairly complete, but that of type $C$ is very fragmentary; of the regular surfaces, only those for which $d=1,2$ 
have been studied in detail (GAETA, 9); of the irregular surfaces, the elliptic surfaces for which $p_{g}=0, p_{a}=-1$ have been described in general terms by Enriques (4) and sub-classes by Campedelit (1) and Conforto-Gherar. DELLI (3). As will appear, a complete classification of the threefolds of linear genus unity requires in the first place a full knowledge of the above types of surface.

2. Some properties of threefolds. - It will be convenient to record here in brief the principal results concerning threefolds which are required in the sequel. (For a short account, see 13; for detailed expositions, SEVERI 20 and 21 may be consulted).

We consider a threefold $V$ which is either non-singular or else is a generic primal of $S_{4}$ (i. e. one endowed with ordinary singularities). If $|A|$ is any surface system on $V$ which is effectively free from base points, and $\left\{A^{\prime} \mid\right.$ is its adjoint system (cutting on $A$ virtual impure canonical curves), the system $|K|=\left|A^{\prime}-A\right|$ is termed the virtual impure canonical system of $V$; we denote its dimension by $P_{g}-1$, where $P_{g}$ is the geometric genus of $V$; if $|K|$ is non-existent as an effective system, we have $P_{g}=0$. Even if $|K|$ is virtual it may happen that, for various values of the positive integer $i$, the systems $|i K|$ are effective; such a system is termed the pluricanonical system of index $i$, or $i$-canonical system, and its dimension is denoted by $P_{i}-1$, where $P_{i}$ is the $i$-genus; if, however, $|i K|$ is virtual, we take $P_{i}$ to be zero.

Denoting by $\Omega_{0}, \Omega_{1}, \mathbf{Q}_{2}$ and $\omega$ respectively the virtual grade, virtual curvilinear genus, arithmetic genus and linear genus of $|K|$, we have the relations, due to PANNeLLI,

$$
2 \Omega_{1}-2=3 \Omega_{0}, \quad \omega=4 \Omega_{0}+1 .
$$

The arithmetic genus $P_{a}$ of $V$ is defined, if necessary by making a preliminary projection on to $S_{4}$, by means of a virtual postulation formula, in a way similar to that followed in the theory of surfaces; its value, in terms of the invariants $\Omega_{i}$, is given by SEverI' s relation,

$$
2 P_{a}=\Omega_{0}-\Omega_{1}+\Omega_{2}+4 \text {. }
$$

The number $q_{1}=P_{g}-P_{a}$ is called the tridimensional irregularity of $V$; it is an absolute invariant, whose value may be positive, negative or zero.

A second absolute invariant is the superficial irregularity $q_{2}$; this is the (constant) irregularity of generic surfaces on $V$, i. e. surfaces which can vary in $\infty^{2}$ linear systems with irreducible free characteristic curves. If $q_{1}=q_{2}=0$, $V$ is said to be completely regular.

Given the characters of the system $|A|$, those of $\left|A^{\prime}\right|$ can be deduced from the fact that $\left|A^{\prime}\right|=|A+K|$ : denoting by $n, \pi, p$ the grade, curvilinear genus and arithmetic genus, and by $p^{(1)}$ the virtual linear genus, 
of $|A|$, with an analogons notation for $\left|A^{\prime}\right|$, we find that

$$
\begin{aligned}
& n^{\prime}=4 n-6 \pi+3 p^{(1)}+3+\mathbf{Q}_{0}, \\
& \pi^{\prime}=6 n-9 \pi+4 p^{(1)}+5+\Omega_{1}, \\
& p^{\prime}=p+2 n-3 \pi+p^{(1)}+3+\Omega_{2} .
\end{aligned}
$$

Hence, using SkverI's relation, we obtain

$$
n^{\prime}-\pi^{\prime}+p^{\prime}=p+2 P_{a}-3 .
$$

An important theorem, due to SEvenI, states that, if $A$ is irreducible and non-singular, $\left|A^{\prime}\right|$ cuts on $A$ a linear system whose deficiency cannot exceed $q_{1}+q_{2}$. From this it at once follows that the freedom $r^{\prime}$ of $\left|A^{\prime}\right|$ is at least $p+P_{a}-1$. In the case where $V$ is completely regular, a more precise result can be obtained; using the same reasoning we may show that $r^{\prime}=p+P_{a}-1$ if, and only if, $A$ is regular: in fact, $r^{\prime}=p+P_{a}-1$, where $p$ denotes the geometric genus of $A$.

This provides a limited form of the RIEmann-RocH theorem, applicable only to certain adjoint systems. A more general form of the theorem has been given by B. SEgre (19). Suppose that, with the above notation, $|A|$ is irreducible and at least simply infinite; then, if $i, \delta$ denote respectively the index of speciality of $|A|$ and the deficiency of the system cut on a $A$ by $|K|$, we obtain the inequality

$$
r \geq n-\pi+p-P_{a}+2+i-\left(q_{1}+q_{2}+\delta\right) .
$$

The expression $R=n-\pi+p-P_{a}+2$ is called the virtual freedom of $|A|$.

3. An inequality for $P_{2}$. - It is well known that, for a non-scrollar surface of arithmetic genus $p_{a}$ and absolute linear genus $p^{(1)}$, the bigenus $p_{2}$ in general (1) satisfies the inequality $p_{2} \geq p_{a}+p^{(1)}$. We shall now establish an analogous result for threefolds.

Suppose that $P_{g}>0$; and let $|A|$ be any system on $V$ which possesses first and second adjoint systems $\left|A^{\prime}\right|$ and $\left|A^{\prime \prime}\right|$ such that $\left|A^{\prime}\right|$ is irreducible and non-singular. The system $\left|A^{\prime \prime}\right|$ cuts on any fixed $A$ a system $|\mathfrak{Q}|$ of curves which, by virtue of relation $A^{\prime \prime}|=| 2 A^{\prime}-A \mid$, and the fact that $P_{g}>0$, is ampler than the canonical system; hence, by PICARD' s theorem for surfaces, the system $|\mathfrak{Q}|$ (which is possibly incomplete), is regular.

We next calculate the characters $n_{2}$ and $\pi_{2}$ of $|\mathfrak{Q}|$, by means of the relations $n_{2}=\left[\left(2 A^{\prime}-A\right)^{2} A\right],\left[\left(A^{\prime \prime}+A\right) A\right]=\left[2 A^{\prime} A\right]$. From the equations given in $\S 2$ we obtain, after reduction,

$$
n_{2}=5 n-8 \pi+4 p^{(1)}+4, \quad \pi_{2}=3 n-5 \pi+3 p^{(1)}+3 .
$$

(1) The exceptional case is the regular surface for which $p_{g}=p_{i}=1$. The inequality yields no information in the ease where $p_{g}=1, p_{n}=0, p^{(1)}=1$; here it is known that $p_{2}>1$ (ENRIQUes, 4). 
Now the number of conditions to be imposed on $A^{\prime \prime}$ in order that it should contain $A$ cannot exceed $n_{2}-\pi_{2}+p+2=2 n-3 \pi+p^{(1)}+p+3$. Also, by the limited form of the RIEMANN-Roor theorem for adjoint systems, the freedom of $\left|A^{\prime \prime}\right|$ is at least $p^{\prime}+P_{a}-1$. It follows that the bigenus $P_{2}$ satisfies the inequality

$$
P_{2}-1 \geq\left(p^{\prime}+P_{a}-1\right)-\left(2 n-3 \pi+p^{(1)}+p+3\right) .
$$

Again using the previous equations, we have, finally,

$$
P_{2} \geq P_{a}+\Omega_{2} \text {. }
$$

It should be noted that although, unlike the corresponding inequality for surfaces, this result holds whenever $P_{g}>0$, it may nevertheless yield no information; for, as will shortly appear, there exist threefolds for which $P_{a}=1, \Omega_{2}=-1, P_{2}>P_{g}>0$.

A similar inequality for $P_{i}$ may be established by considering the curve system cut by the $i$ th adjoint system $\left|A^{(i)}\right|$ on $A$. For, since $A^{(i)} \equiv i A^{\prime}-(i-1) A$, the grade $n_{i}$ and genus $\pi_{i}$ of the system are given by the formulae $n_{i}=\left[\left(i A^{\prime}-(i-1) A\right)^{2} A\right], \pi_{i}=\left[\left(i A^{\prime}-(i-1) A\right) A\right]$. A simple calculation shows that

$$
\begin{aligned}
& n_{i}=i^{2}\left(p^{(1)}-1\right)-2 i(i-1)(2 \pi-2-n)+(i-1)^{2} n \\
& \pi_{i}=i p^{(1)}+\left(\begin{array}{l}
i \\
2
\end{array}\right)\left(p^{(1)}-1\right)-(i-1) \pi-i(i-1)(2 \pi-2-n)+\left(\begin{array}{l}
i \\
2
\end{array}\right) n .
\end{aligned}
$$

The number of conditions to be imposed on $A^{(i)}$ in order that it should con. tain $A$ cannot exceed $n_{i}-\pi_{i}+p+2$; while the freedom of $\left|A^{(i)}\right|$ is at least $p^{(i-1)}+P_{a}-1$, where $p^{(i-1)}$ denotes the arithmetic genus of $A^{(i-1)}$. Thus

$$
P_{i}-1 \geq\left(p^{(i-1)}+P_{a}-1\right)-\left(n_{i}-\pi_{i}+p+2\right) .
$$

Substituting for $n_{i}$ and $\pi_{i}$, we obtain, after reduction,

$$
\begin{aligned}
P_{i}-1 \geq & \left(p^{(i-1)}+P_{a}-1\right)-\frac{1}{2} i(i-1) p^{(1)}+(i-1)(2 i-1) \pi- \\
& -\frac{1}{2}(i-1)(3 i-2) n-\frac{1}{2} i(3 i-5)-p-2 .
\end{aligned}
$$

The formulae for the virtual characters of the pluricanonical system $|i K|$, which will be required later, are deducible from the formulae of SEVERI (20) for those of any assigned multiple of a given linear system; with an obvious notation, we have the results

$$
\begin{aligned}
& \Omega_{0}{ }^{(i)}=i^{3} \Omega_{0}, \Omega_{1}{ }^{(i)}=i^{2}\left(\Omega_{1}-1\right)+i^{2}(i-1) \Omega_{0}+1, \\
& \Omega_{2}{ }^{(i)}=\left(\begin{array}{l}
i \\
3
\end{array}\right)\left(\Omega_{0}-1\right)+\left(\begin{array}{l}
i \\
2
\end{array}\right) \Omega_{1}+i \Omega_{2}+\left(\begin{array}{c}
i-1 \\
3
\end{array}\right) .
\end{aligned}
$$

The virtual linear genus $\omega^{(t)}$ of the surface $i K$ is deducible from a general formula of B. SEgRe (18); this gives

$$
\omega^{(i)}=1+i(\omega-1)+i(i-1)(i-3) \Omega_{0}+4 i(i-1)\left(\Omega_{1}-1\right) .
$$


4. The product of a curve and a surface. - Consider the threefold $V$ which maps the pairs of points of an irreducible non-singular curve $\mathcal{C}$ and an irreducible non-singular surface $F$; we suppose that $\mathcal{C}$ bas genus $\pi$ and that $F$, which is free from exceptional curves of the first species, has genera $p_{g}, p_{a}$ and absolute linear genus $p^{(1)}$.

SEverI (20) has shown that, on $V$, a virtual canonical surface $K$ satisfies the equivalence

$$
K \equiv(2 \pi-2) F_{0}+C_{0},
$$

where the $2 \pi-2$ surfaces $F_{0}$, corresponding to points of a canonical set on $\mathfrak{C}$, are each obtained by fixing a point of $\mathfrak{C}$ and allowing its companion to vary on $F$; and where $C_{0}$ denotes the product of $\mathcal{C}$ with a virtual pure canonical curve of $F$.

Severi has further shown that the invariants of $V$ are given by the formulae $\left({ }^{2}\right)$

$P_{g}=\pi p_{g}, \quad P_{a}=(\pi-1) p_{a}+\pi, \quad \Omega_{0}=6(\pi-1)\left(p^{(1)}-1\right), \quad q_{2}=p_{g}-p_{a}+\pi$.

In the applications to be made of these results in cases where $K$ is effective, we require the canonical system to be pure, i. e. free from exceptional surfaces. It is clear that, if $F$ contains an exceptional curve of the first species, this will give rise to an analogous surface on $V$; but it is not known in general whether the purity of $|K|$ is a consequence of the freedom of $F$ from such curves $\left({ }^{3}\right)$. In the illustrations which follow this is certainly the case.

In connection with $\$ 3$, we may now show that there exist threefolds with effective canonical systems for which $P_{a}=1, \Omega_{z}=-1, P_{z}>1$. Consider first the case where $\mathcal{C}$ is elliptic and where $F$ is a regular surface with an elliptic canonical curve $\left(p_{g}=p_{a}=p^{(1)}=1, p_{2}=2\right)$; here $P_{g}=P_{a}=q_{2}=1$, and the canonical surface is an irregular hyperelliptic surface, the product of two elliptic curves, so that $\Omega_{z}=-1$. In this case there is a pencil of bicanonical surfaces.

More generally, suppose that $F$ possesses a canonical system which is compounded of a pencil (rational or irrational) of elliptic curves; here we have $P_{g}=p_{g}, P_{a}=1, q_{\mathrm{a}}=p_{g}-p_{a}+1$, while, as before, $\Omega_{2}=-1$. In the case where $p_{g}>1$, the characteristic system of $|K|$ is compounded of a congruence of elliptic curves.

(2) The first of these has been obtained by GAETA (10) from geometrical reasoning; the original proof was transcendental.

(3) GaETA (10) has conjectured that a similar result holds for the product of any number of given varieties.

It may be noted that, even for a threefold with $P_{g}>0$. it is not known whether the canonical system can always be cleared of exceptional surfaces: see Severi (21). 
5. 'Threefolds with linear genus unity. - Suppose now, in the first place, that $V$ possessess an effective pure canonical system $|K|$ of order greater than zero. If $A$ is any irreducible non-singular surface of $V$, it follows that $A^{\prime} \mid$ must eut the virtual pure canonical system on $A$. This remark applies also to $K$ itself, for which $\left|K^{\prime}\right|=|2 K|$; hence, if $P_{g}>1, K$ certainly possesses an effective canonical system (which may be of order zero), except possibly when $|K|$ is compounded of a pencil.

We shall make the assumption that, if the generic $K$ is irreducible, it is simple; in this case the computed value of $\omega$ in $\S 2$ is the absolute linear genus of $K$; and we shall take this to be unity. It then follows from $\S 2$ that $\Omega_{0}=0, \Omega_{1}=1$. If the generic $K$ is reducible, we suppose for the present that is has no fixed part; the condition $\omega=1$ then shows that $|K|$ must be compounded of a pencil (rational or irrational) without base points. (Later, in $\S 11$, we shall relax this assumption).

Suppose, in the second place, that $K$ is the null surface; then its virtual characters being in fact $n=0, \pi=1, p=-1, \omega=1$, we see that this leads to another possible type.

Next, suppose that $|K|$ is virtual but that $|i K|$ is effective for some positive value of $i$; if, in regard to this system, we make assumptions similar to those already made for $|K|$, we see that the equations in $\& 3$ then give the values previously obtained for the invariants $\Omega_{i}$ and $\omega$.

We have still to consider the possibility that $P_{i}=0$ for all $i \geq 1$. The question of defining $w$ in this case has yet to be settled; by analogy with CASTELNUOVO's work on the absolute linear genus of a scrollar surface, we may expect it to be connected with the problem of eliminating the exceptional surfaces from a given threefold. It is not known at present whether this can always be achieved (for exceptional surfaces « of the first kind»), although there is a restricted class of threefold, having $P_{i}=0$, for which the elimination is possible (\$ 7). We shall therefore limit the consideration of this case to particular examples.

Corresponding to the surfaces considered in $\$ 1$, there will thus be four main types of threefold; for, in the present case, two types arise by analogy with class $C$.

I. Parallel to the elliptic scrollar surfaces we have threefolds whose canonical and pluricanonical systems are all virtual. Presumably there exist both regular and irregular types; by making a provisional assumption, we are enabled to obtain examples of these from the results of $\S 4$.

II. The next class consists of threefolds possessing isolated (pure) canonical and pluricanonical surfaces (possibly of order zero); for these, $P_{i} \leq 1$. If $K$ has order greater than zero, its absolute linear genus is 1 , and the virtual grade and curvilinear genus of $|K|$ are 0 and 1 respectively. From the formulae in $\S 3$ it follows that the system $|i K|$ coincides with $|K|$, for 
all values of $i$, if, and only if, $\Omega_{z}=-1$; hence, by SEveri's relation, $P_{a}=1$. If, instead, $K$ is the null surface, we again find from Severi's relation that $P_{a}=1$. These results are in contrast with those of $\S 1$; for surfaces of class $B$ can have arithmetic genus \pm 1 , while those which possess a single canonical curve of effective genus 1 must have at least a pencil of bicanonical curves.

The two types analogous to class $C$ possess at least $\infty^{1}$ canonical or pluricanonical surfaces of some index, except possibly when $P_{g}=0$.

III. In the first case the effective canonical or pluricanonical systems are compounded of a pencil (rational or irrational) of surfaces of zero virtual grade. Here the eharacteristic cnrves of the system or systems in question have order zero. When $P_{g}=0$, the effective pluricanonical systems may also have order zero.

IV. In the second case the characteristic curves have order greater than zero; hence they must be elliptic. Here the generic canonical or pluri. canonical surface (as the case may be) is irreducible, but the corresponding characteristic system is compounded of a congruence of the first order. Each surface in question is generated by a pencil, rational or irrational, of elliptic curves.

These four classes of threefold have been obtained on the hypotheses stated above; in the present state of the theory it does not seem possible to say whether they include all types: thus there might conceivably be a single canonical surface with multiple components, perhaps comprising irremovable exceptional surfaces. But no such cases are known at present.

There is another kind of exceptional surface which can arise in the present work: this is the so-called singular exceptional surface, which is transformable to an unassigned base point or base curve of the canonical system $|K|$. In the present case, where $\omega=1$, the relation $\omega=4 Q_{0}+1$ shows that the virtual grade of $|K|$ is zero; if, however, there are unassigned base elements, the effective grade is different from zero.

In order to obtain examples of threefolds possessing this feature and belonging to class III or IV, it suffices to consider the product $V$ of an elliptic eurve $\mathcal{C}$ and a surface $F$ whose canonical system $|\mathcal{H}|$ has unassigned base points; surfaces of this type are, of course, well known. Each such base point gives rise to an elliptic earve which is an unassigned base curve of $K \mid$; evidently $K$ has a canonical system which is compounded of an irrational pencil of elliptic curves, so that in fact $\omega=1$. $V$ then belongs to class III or to class IV, according as the freedom of $|\mathcal{H}|$ is equal to or greater than unity.

In what follows we shall assume that the canonical systems $|K|$ under discussion are free from unassigned base elements; thus in every case where there are no assigned base points, the effective grade of $|K|$ is zero. 
6. Analogies with the theory of curves and surfaces. - The majority of surfaces with absolute linear genus unity contain pencils of elliptic curves; and, as will appear, many of the threefolds having $\omega=1$ possess canonical surfaces which contain such peneils.

A surface which contains a net of elliptic curves is, however, rational or elliptic scrollar and it is remarkable that a threefold which contains a net of surfaces of the above type is in most cases unirational, or birationally equivalent either to a cone projecting one of these surfaces, or to the product of a rational surface and an elliptic curve: the exceptional cases occur when the characteristic system of the net is compounded of elliptic eurves, or when the surfaces contain more than one pencil of elliptic curves, and it is impossible to separate the pencils rationally (cf. 15).

A different analogy between certain classes of curves, surfaces and threefolds, which proves useful in problems of construction, is as follows. To begin with, consider an elliptic curve; this has a canonical series of order zero, and the sum of two or more such curves, no two of which intersect, is also a (virtual) elliptic curve. Again, by taking a multiple elliptic curve we can represent other elliptic curves on it without the intervention of branch points.

Consider next a surface with $p_{a}=-1$; this has an EnRIQUes series of order $12\left(p_{a}+1\right)=0$; and any number of such surfaces, without common points, are additive in the previous sense. Further, as Enriques has shown, all the surfaces for which $p_{g}=0, p_{a}=-1$ cin be represented on a multiple elliptic scroll whose branch curve consists of a set of prime sections belonging to a pencil; and, more generally, the paraelliptic surfaces (which contain elliptic pencils of elliptic curves) may be represented in a similar manner. Finally, we may note the results, due to ENRIQUes and SEVERr (5), that a special type of irregular hyperelliptic surface, containing two (elliptic) pencils of elliptic curves, may be represented on a multiple elliptic surface, free from branch curves; and that a general JACOBI surface may be represented on a double KUMMen surface, likewise free from branch curves.

The threefolds of genus $P_{a}=1$ have what we may call a SEgre series (B. SEgRE, 18) of order $24\left(P_{a}-1\right)=0$. Severr' s formula for the arithmetic genus of a composite threefold shows that such varieties are likewise additive if they have no common points. Moreover, it will be seen that one can construct ample elasses of $V_{3}$ for which $P_{a}=1, \omega=1$, by quite simple methods.

A more detailed investigation will probably bring out further analogies with the preceding theories.

There are. however, certain respects in which the theories of surfaces and threefilds differ fundamentally. Thus, the arithmetic genus $p_{a}$ and the absolute linear genus $p^{(1)}$ of a surface are independent, since NoETHER' s relation $12 p_{a}+9=p^{(1)}+i$, introduces a third invariant 1 ; on the other hand, 
SEveri's relation for threefolds expresses $P_{a}$ in terms of the invariants $\Omega_{i}$, so that, in the case we are considering, where $\Omega_{0}=0, \Omega_{1}=1$, we have $2 P_{a}=\Omega_{2}+3$. Here the ZeUTheN-SEGRE invariant $I$ is independent of the above characters.

In the classification of threefolds, an important part is played by the superficial irregularity $q_{2}$; for the variety considered in $\$ 4$, this is certainly independent of the other invariants, and is so, presumably, for threefolds in general.

7. Some threefolds of class $I$. - As already remarked, in the case of a threefold for which $P_{g}=P_{i}=0$, it has still to be shown that an absolute linear genus $\omega$ can be defined. If $V$ is the product of a curve $C$ (of genus $\pi$ ) and a surface $F$ with relative linear genus $p^{(1)}$. it follows that the character $\omega=4 \Omega_{0}+1$, where $\Omega_{0}$ is given by the appropriate formula in $\S 4$, attains its maximum value with $p^{(1)}$, i. e. when $p^{(1)}$ is the absolute linear genus of $F$. For this reason we shall define $\omega$ as the value obtained by taking $p^{(1)}$ to be the absolute linear genus of $F$. We thus have $\omega=1$ whenever $p^{(1)}=1$, or $\pi=1$.

Suppose now that $F$ has invariants $p_{y}=p_{a}=0, p^{(1)}=1$, (so that $p_{z}>0$, and that $\mathcal{C}$ is rational ; then it may be shown that $V$ is completely regular, with $P_{g}=P_{i}=0(\mathbf{1 4})$.

GAETA (9) has established the existence of a simply-infinite series of such surfaces $F$, having divisors $\sigma=2^{r}(r=1,2,3, \ldots)$. It may be proved that the corresponding threefolds $V$ have divisors $\sigma=2^{r}$, from which it follows that they are birationally distinct, and that they cannot be birational; and it may further be shown that they are not even unirational (14).

These threefolds are in a sense analogous to the elliptic scrollar surfaces. $A$ different analogy is provided by the product $V$ of an elliptic curve $\mathcal{C}$ and a surface $F$ with invariants $p_{g}=p_{i}=0$. In this case we have $P_{g}=0, P_{\alpha}=1$, $q_{2}=1-p_{a} ;$ and since, by $\S 4, K \equiv C_{0}$, while $F$ has no pluricanonical system of any index, it follows that $P_{i}=0$. If, in particular, we take $F$ to be rational, then $V$ is generated by an elliptic pencil of rational surfaces: in all other cases $F$ is scrollar, and $V$ contains a congruence, of the first order, of rational curves.

Completely regular threefolds of genera and plurigenera zero, birationally distinct from the above types (since they all have divisor unity) are already well known. (FANO, 6, $\boldsymbol{7}$ ); it may throw light on the present problem if we recall some of the results which have been obtained for them.

Among these threefolds, which are endowed with canonical curve sections, there is a finite number which contain only complete intersections with primals (16); since these are free from exceptional surfaces, it is certainly possible to eliminate such surfaces in some cases. Favo has shown that, subject to a certain working hypothesis, the maximum freedom of the linear 
systems of surfaces of genera and plurigenera unity which each such threefold contains is equal to $-\left(\Omega_{2}+1\right)$; and he has proposed (8) to introduce this number, which is obviously an absolute invariant, into the study of threefolds of genera and plurigenera zero. It would be interesting to discover whether the corresponding character is equal to $-\left(\Omega_{2}+1\right)$ for each of the completely regular threefolds considered above.

8. Threefolds of genera $P_{i} \leq 1$. - To begin with, suppose that $V$ is a threefold of class II, with a pure canonical surface of order zero; in this case $P_{g}=P_{i}=1$, and, as stated in $\S 5, P_{a}=1$. Thus in all such cases $q_{1}=0$, i. e. $V$ can be at most superficially regular.

For any given linear system $|A|$ on $V$, we must have $\left|A^{\prime}\right|=|A|$. Hence, if the system is free from base points, its grade $n$ and curvilinear genus $\pi$ are connected by the relation $n=\pi-1$; moreover, $\pi$ is the absolute linear genus of $A$. In particular, if $A$ belongs to a pencil free from base points, it must possess a canonical curve of order zero; a threefold containing such a pencil is analogous to a surface with invariants $p_{g}=p_{i}=1$, which contains a pencil of elliptic curves; and, like the latter, is presumably a sub-species when it is completely regular.

Suppose that, for the present, $V$ is completely regular; then the characteristic system of any complete system $|A|$ contained in it must be complete; hence the freedom of $|A|$ is equal to the genus $p$ of $A$. In particular, the prime sections of $V$ are regular canonical surfaces of some genus $p$, and $V$ is normal in $S_{p}$.

The problem of classifying the completely regular types depends, therefore, in the first instance upon that of elassifying the regular canonical surfaces. Apart, however, from the two simply-infinite families about to be described, all the canonical surfaces so far studied consist of isolated examples (ENRTQUES, 4), so that no general theory is available for the present purpose.

The first of these families, which was discovered by NOETHER, comprises the surfaces for which $\pi$ has the minimum value $2 p-3$ with respect to $p$; in this case the canonical curves are hyperelliptic- We shall now show that there exists only a finite number of corresponding threefolds having base number unity.

Suppose that $V$ contains a system $|A|$ whose characters $\pi$ and $p$ satisfy the relation $\pi=2 p-3(p \geq 3)$; then the characteristic curves of $|A|$ are hyperelliptic, and the characteristic series is compounded of an involution $I_{2}$. Thus $V$ may be mapped on a double threefold $W$ whose eurve sections are rational, and which is therefore one of the following types:

(1) a space $S_{3}(p=3)$;

(3) a planar threefold $W^{p-2}(p=5,6, \ldots)$;
(2) a general quadric $W^{2}(p=4)$;

(4) a VeRoNESE cone $W^{4}(p=6)$. 
We readily see that, in (1), the branch surface is a general octavic $F^{8}$, and that, in (2), it is a $F^{12}$ ent on $W^{2}$ by a sextic primal. As regards (3) we observe that $W$ contains a pencil of generating planes, without base points, which must correspond to surfaces for which $p=1, \pi=1$; hence. if we assume that the latter are of general type, it follows that the branch surface $F$ on $W$ meets the generating planes in sexties which in general are non-singular. We are thus led to a particular case of the threefolds examined later in $\$ 10$, where $p=2, d=1:$ if $R$ denotes a generating plane of $W$, and $D$ a directrix surface of minimum order (meeting $R$ in a line) we find that $F$ is of the form $6 D+8 R, 6 D+6 R, 6 D+4 R$, according as $|D|$ has frcedom 0,1 , or 2 . In (4) the net of double quadric cones with vertex at the vertex of $W$ must map surfaces for which $p=2, \pi=1$, whence we see that the branch surface is cut by a quintic primal passing simply through the vertex. Evidently, then, only in cases (1) and (2) has $V$ base number unity.

The second family of surfaces, discovered by CASTELnuovo, consists of those for which $\pi$ assumes the minimum $3 p-6$ with respect to $p$, on the hypothesis that the characteristic series of the canonical system (assumed to be irreducible) is simple. The threefolds having such surfaces for prime sections hare been investigated by d'ORGEVAL (12); they comprise only a finite number of birationally distinct general types.

It is well known that the most general regular surfaces for which $p_{g}=p_{i}=1$, depend on 19 moduli, and have base number unity: in contrast with this, most of the threefolds of class II which have so far been constructed have base number exceeding unity, and it is possible that the number of birationally distinct threefolds of this class which have base number unity is finite. Again, it may be noted that the number of moduli of these threefolds, as computed from B. SeGRE's provisional formula (19), is not constant.

The superficially irregular threefolds of class II depend for their speci. fication on the irregular canonical surfaces, about which little is known at present. There is, however, one familiar, type, the Abelian variety of genus 3 which, in its most general form, is birationally equivalent to the threefold which maps the triads of points of a carve of genus 3 (LEFscheTz, 11; SEVERI, 22). This variety has base number unity and superficial irregularity 3 ; it is analogous to the JACOBr (hyperelliptic) surface of general type, which maps the pairs of points of a curve of genus 2. Just as there exist special forms of this surface, with base number greater than unity, and containing pencils of elliptic curves, so we find particular forms of the Abelian threefold with similar properties: consider, for example, the product of an elliptic curve and a hyperelliptic surface for which $p_{g}=1 \quad p_{a}=-1$; here we have $P_{g}=P_{i}=P_{a}=1, q_{2}=3$, as before, but the threefold contains a tongruence, of the first order, of elliptic curves which is not present in the general case.

Another superficially irregular type is represented by the product of an 
elliptic curve and a regular surface for which $p_{g}=p_{i}=1$; in this case $P_{g}=P_{i}=P_{a}=1$, while $q_{2}=1$.

We have next to consider the possibility that $V$ may possess a pure canonical surface $K$, of order greater than zero, such that all the $i$-canonical systems coincide with $K$, counted $i$ times. In the theory of surfaces the analogous case cannot oceur; for it is known that, if a surface has a canonical curve of order greater than zero, it must have at least a pencil of bicanonical curves.

The formulae for $|i K|$ given in $\S 3$ show that, in the present case $\Omega_{0}=0$, $\Omega_{1}=1, Q_{2}=-1$; hence $P_{a}=1$, and so $q_{1}=0$. Again, since $K^{\prime} \equiv 2 K$, we see that $K$ possesses a canonical curve of order zero; thus, if the surface is irreducible, it is hyperelliptic.

Threefolds of genus $P_{g} \geq 1$, which possess canonical surfaces of this type, have some interesting properties which we shall consider briefly. Suppose that $|A|$ is an irreducible non-singular system on such a threefold $V$, cutting $K$ (supposed irreducible) in a system $|\mathfrak{Q}|$ of curres of some genus $\rho \geq 1$. (If $K$ were the null surface, we should have $\rho=1$, for every system $|A|)$. The grade $v$ of $|\mathfrak{G}|$ is equal to $2 p-2$, while, for $\rho>1$, the freedom of the system (completed if necessary) is $\rho-2(\S 9):$ if $\rho=1,|\mathfrak{Q}|$ is compounded of an elliptic pencil.

Consider now an adjoint $A^{\prime}=A+K$, of $A$, cutting $A$ in curves of the virtual pure canonical system; since this system has genus $p^{(1)}$ and grade $p^{(1)}-1$, we have the relations $p^{(1)}=\left[A A^{\prime}\right], p^{(1)}-1=\left[A A^{\prime 2}\right]$.

As in $\S 2$, we write $\left[A^{3}\right]=n,\left[A^{2}\right]=\pi,[A]=p ;$ then, since $[A K]=\rho$, $\left[A^{2} K\right]=v=2 \rho-2$, we obtain

$$
p^{(1)}=[A(A+K)]=\pi+p+\nu-1, \quad p^{(1)}-1=\left[A(A+K)^{2}\right]=n+2 v .
$$

Subtracting the second of these equations from the first, we have the relation

$$
\pi-n=p .
$$

Next, writing $\left[A^{\prime} K\right]=\rho^{\prime},\left[A^{\prime}\right]=p^{\prime}$, with a similar notation for the successive adjoints, we have $\left[A^{\prime} K\right]=[(A+K) K]=[A K]+\left[K^{2}\right]+[A K]-1$. Hence $\rho^{\prime}=\rho$, and thus $\rho=\rho^{\prime}=\rho^{\prime \prime}=\ldots$. Now $K$ is a surface with divisor nnity, for it has a canonical curve of order zero; hence $|A|,\left|A^{\prime}\right|,\left|A^{\prime \prime}\right| \ldots$ all cut $K$ in curves belonging to the same continuous system.

Again, $p^{\prime}=\left[A^{\prime}\right]=[A+K]=[A]+[K]+[A K]=p+p-1$. So $p^{\prime \prime}=p+$ $+2(\rho-1)$, and, generally, $p^{(i)}=p+i(\rho-1)$.

So far we have made no assumptions concerning the plurigenera of $V$. If we substitute for $\pi, p^{(1)}$, and $p^{(t)}$ in the inequality for $P_{i}$ given in $\S 3$, we merely obtain the result $P_{i} \geq 0$. However, using the theory of continuous systems, we can show that, if $P_{i}=1$, then $V$ must be completely regular. For we have seen that $q_{1}=0$ : suppose then that, if possible, $q_{2}>0$. In that case it is known (B. SEgRE, 19) that any sufficiently general 
linear system of surfaces on $V$ must belong to a continuous system composed of $\infty q_{2}$ linear systems. Assuming that $\left|A^{\prime}\right|$ is such a linear system, we deduce that it belongs to a continuous system $\left\{\overline{A^{\prime}} \mid\right.$ of para-adjoints; hence there exists a continuous system of paracanonical surfaces $\bar{A}^{\prime}-A$, which, since $P_{g}=1$, must be a pencil, necessarily irrational. It follows that $V$ possesses at least a pencil of bicanonical surfaces, i. e. $P_{2}>1$.

More generally, we may envisage the possibility that $P_{i}=0(i<j)$, $P_{i}=1(i \geq j)$. Here, again, we deduce that $\Omega_{2}=-1$ and hence that $P_{a}=1$; also, as before, we can show that $q_{2}=0$. For consider a general system $\left|A^{(j)}\right|$ of $j$-adjoint surfaces; if $q_{2}>0$, this will belong to a continuous system $\left|\overline{A^{(j)}}\right|$ of para-j-adjoints, so that. by the above reasoning, there must be at least a pencil of $2 j$-canonical surfaces. However, no examples of this or the preceding type of threefold are known.

9. Threefolds of class III : the case $\sigma=1$. - Consider next the case where $V$ has a pure canonical system $|K|$ compounded of a pencil which, for the present, we shall assume to be rational. We shall also suppose, in the present section, that the pencil $|C|$, which is effectively free from base points $(\S 5)$, contains no redueible and, also, no multiple members. There then subsists an equivalence $K \equiv\left(P_{g}-1\right) C$, where we may assume $\left({ }^{4}\right)$ that $P_{g}>1$; thus all the pluricanonical systems are effective, and are compounded of $|C|$. In this case, evidently, $V$ has divisor $\sigma=1$.

We have first to determine the characters of $C$. To begin with, we observe that $C$ has a pure canonical curve of order zero $(5)$; this is an immediate consequence of the relations $C^{\prime} \equiv C+K \equiv P_{g} C$. Hence $C$ has absolute linear genus unity, and is either regular, with $p_{g}=p_{i}=1$, or irregular hyperelliptic, with $p_{g}=p_{i}=1, p_{a}=-1$.

Now the invariants $\Omega_{0}, \Omega_{1}, \Omega_{2}$ have the respective values 0,1 , $\left(P_{g}-1\right)\left(p_{a}+1\right)-1$; substituting these numbers in SeverI's relation, we obtain $2\left(P_{a}-1\right)=\left(P_{g}-1\right)\left(p_{a}+1\right)$. From this we conclude that

(1) if $O$ is regular, then $P_{g}=P_{a}$;

(2) if $C$ is irregular hyperelliptic, then $P_{a}=1$, while there is no restriction on the value of $P_{g}$.

Conversely, we see that, if $P_{g}=P_{a}, C$ must be regular; and that, if $P_{a}=1\left(P_{g}>1\right), C$ must be irregular.

Suppose now that $C$ is regular; then the most general type of such surface - which is what we shall consider here - is characterized by the genus $p(\geq 2)$ of the curve $\mathcal{C}$ which forms the base on $C$. This curve belongs

${ }^{(4)}$ The case $P_{q}=1$ may be excluded as of minor interest, since it belongs to class II.

(5) If we allow $|K|$ to have unassigned base curves, the equivalence $K^{\prime} \equiv 2 K$ shows that they must be elliptic, forming part of the eanonical system of $C$; thus $C$ has a cano. nical system compounded of a pencil of elliptic curves. 
to a system $|\mathfrak{C}|$ of grade $2 p-2$ and freedom $\rho$; any other curve system $|\mathfrak{D}|$ on $C$ is of the form $|d \mathfrak{e}|$, where $d>1$, and its virtual genus, grade and freedom are $\rho_{1}, 2 \rho_{1}-2$. and $\rho_{1}$ respectively, where $\rho_{1}=d^{2}(\rho-1)+1$.

Similar results hold in the case where $C$ is hyperelliptic of the most general type (EnRIQUes-Severi, 5). Thus the base on $C$ is furnished by a single curve $\mathcal{C}$, of genus $p \geq 2$, while, with the above notation, any curve system $|d e|$ on $C$ has genus $\rho_{\text {, }}$ and grade $2 \rho_{1}-2$; the freedom of the system is, however, $\rho_{1}-2$.

In conclusion, then,

If the effective pure canonical system of $V$ is compounded of a rational pencil of surfaces, effectively free from base points, and con aining no reducible or multiple members, then all the pluricanonical systems are effective, and are compounded of this pencil. The surfaces of the pencil are either regular of genera and plurigenera unity, or irregular hyperelliptic: in the first case $P_{g}=P_{a}$, while in the second, $P_{a}=1$.

10. Classification of $V$. - In either of these cases, a basis for the classification of $V$ is obtained by considering the surfaces $A$ which cut the generic $C$ in curves $d \mathcal{C}$, where $d$ is a minimum. We call these $d$-secant surfaces, and we suppose they are non-singular; $d$ may be termed the determinant of $V$. We then consider the system (or systems) $|A|$ of $d$-secant surfaces of minimum virtual grade. Evidently the freedom $r$ of the complete system $|A|$ cannot exceed $\rho_{1}$, or $\rho_{1}-2$, according as $C$ is regular or irregular, for

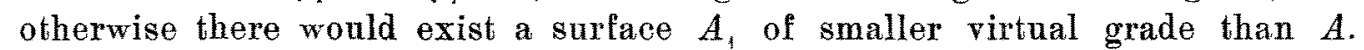

As in $\S 2$, we denote the virtual characters of $|A|$ by $n, \pi, p$, respectively, writing $\left[A^{3}\right]=n,\left[A^{2}\right]=\pi,[A]=p$; evidently $n, \pi, p, r$ are absolute invariants of $V$, since, even when $r=0, A$ cannot be an exceptional surface. We also denote by $p^{(1)}$ the virtual linear genus of $A$.

Consider an adjoint $A^{\prime}=A+K$, of $A$; since this cuts on $A$ curves of the virtual pure canonical system, of grade $p^{(1)}-1$, we have the relations $p^{(1)}-1=\left[A A^{\prime 2}\right], p^{(1)}=\left[A A^{\prime}\right]$. Substituting $A^{\prime}=A+K$, and using the results $[A C]=p_{1},\left[A^{2} C\right]=2 p_{1}-2,\left[A C^{2}\right]=0$, we obtain

$$
\begin{aligned}
p^{(1)}-1 & =n+4\left(P_{g}-1\right)\left(\rho_{1}-1\right), \\
p^{(1)} & =\pi+3\left(P_{g}-1\right)\left(\rho_{1}-1\right) .
\end{aligned}
$$

Now $|A|$ is certainly non-special; hence its virtual freedom $R$ is given by

$$
R=n-\pi+p-P_{a}+2=p-P_{a}+1-d^{2}(\rho-1)\left(P_{g}-1\right) .
$$

In virtue of the RIEMANN-RocH theorem $(\S 1)$, all that can in general be asserted is that, if $r>0, r \geq R-\left(q_{1}+q_{2}+\delta\right)$. If however, we assume that $V$ is completely regular, with $P_{g}=P_{a}=P$, so that $C$ also is regular, 
we see that $|K|$ cuts a complete system on $A$, i. e. $\delta=0$. We then have $\left(^{6}\right)$

whence

$$
r \geq n-\pi+p-P+2
$$

$$
r \geq p-(P-1)\left\{1+d^{2}(p-1)\right\} \text {. }
$$

Here, as already remarked, $r$ may assume the values $0,1, \ldots, d *(\rho-1)-1$. For any given value of $r$, this inequality provides an upper limit for $p$.

In his discussion of the regular surfaces of absolute linear genus unity and determinant 1 or 2, GAETA (9) has shown that the most general types of such surfaces (i. e. with the maximum numbers of moduli) are those for which the curve systems analogous to $|A|$ are regular. It would be interesting to see whether a like conclusion is valid in the present instance.

With regard to the characters of $A$ we observe that, except possibly when $r=0, A$ must have geometric genus $p_{g}>0$. Again, when $r=1,|A|$ cuts a pencil of curves on the generic $C$ which, whether $C$ is regular or not, has positive grade, which means that $|A|$ is endowed with a base curve.

Suppose now that $A$ is regular (as will certainly be the case when $V$ is superficially regular and $A$ generic); if its genus $p$ is greater than zero, we then have the inequality $p^{(1)} \geq 2 p-3$, the minimum value of $p^{(1)}$ being attained when the canonical curves of $A$ are hyperelliptic. The maximum value of $p^{(1)}$ is given by Rosenblat's' s inequality (ENRIQUES, 4 ), $p^{(1)} \leq 16 p+27$ ).

In order to obtain a representation of $V$ in the case where the threefold is completely regular, we consider the system $\left|A^{\prime}\right|=|A+K|$; except possibly when $r=0$, this is irreducible, since it has no fixed part and is not compounded of a pencil. Its characters $n^{\prime}, \pi^{\prime}, p^{\prime}$ are given by the equations in $\S 2$, where we must put $\Omega_{0}=0, \Omega_{1}=1, \Omega_{2}=2 P-2$, and where $P_{g}=P_{a}=P$. We know that the freedom $r^{\prime}$ of $\left|A^{\prime}\right|$ is at least $p+P-1$, and is precisely this if, and only if, $A$ is regular. Provided $r^{\prime} \geq 3$, we can map $\left|A^{\prime}\right|$ on the prime sections of a threefold $W$ which will be a simple or multiple model of $V$; for although the characteristic series of $\left|A^{\prime}\right|$ may be compound, it is easily seen that the characteristic system must be simple: this is a consequence of the fact, immediately deducible from the preceding equations, that $n^{\prime}>0$.

We illustrate these ideas by examining the case $p=2, d=1$; here we have $r=0,1$, or 2 . By means of the net $|\mathfrak{C}|$ contained in $C$, which sets up a rational involution $I_{2}, C$ may be transformed into a double plane; at the same time, each surface $A$, which contains a pencil $|\mathcal{C}|$, will be transformed to a minimal directrix $D$ of the system $|R|$ of planes which maps $|O|$; these generate a threefold $W$, having rational curve sections, and of order

(6) We require here the extension of the RIEMANN-Roch theorem to the case of an isolated non-singular surface on a completely regular threefold; this is easily established by the same reasoning as that employed in the general case. 
$3 m-2,3 m-1$, or $3 m$, according as $r=0,1$, or 2 ; in the first two cases we may take $m \geq 2$, and in the third, $m \geq 1$.

The main facts concerning $V$ are now readily determined by using the representation of $W$ on $S_{3}$, in which prime sections, of $W$, of order $N$, say, are mapped by scrolls of order $N$, having an $(N-1)$-fold base line, and $N-1$ simple base lines incident to it. Thus, we see that the system $|D|$ has virtual grade $-2,-1$, or 0 , according as $r=0,1$, or 2 , and that, in the second case, $|D|$ possesses a rational base curve which is unisecant to $R$. Hence, in the previous notation, the corresponding values of $n$ are $-4,-2$, and 0 ; and, in the second case, $|A|$ has a base curve bisecant to $R$.

Again, from the representation, we find that the branch surface $F$ on $W$ is given by $F \equiv 6 D+2 \mu R$, where, in the three cases, $\mu$ takes the respective values $P+3, P+2, P+1$. Assuming that $F$ is non-singular, we then proceed to calculate the remaining characters of $|A|$, obtaining the following results :

$$
\begin{aligned}
& r=0: \quad n=-4, \quad \pi=P-4, \quad p=2 P-2, \quad p^{(1)}=4 P-7 . \\
& r=1: \quad n=-2, \quad \pi=P-2, \quad p=2 P-1, \quad p^{(1)}=4 P-5 \text {. } \\
& r=2: \quad n=0, \quad \pi=P, \quad p=2 P, \quad p^{(1)}=4 P-3 \text {. }
\end{aligned}
$$

In all cases, therefore, we have $p^{(1)}=2 p-3$; and we find that the system $|A|$ is regular.

11. The case $\sigma>1$. - The threefolds just considered form in reality only a restricted sub-species of class III, inasmuch as their plurigenera are all given by the formula $P_{i}=i\left(P_{g}-1\right)+1$. We now show that a more extensive class may be constructed by a process exactly akin to that described by ExRIQUes (4) for the regular surfaces of absolute linear genus 1 and determinant $d>1$.

We shall suppose that the pure canonical system $|K|$, effective or virtual, is compounded of a rational pencil $|C|$. without base points, which now contains a certain number $S(\geq 1)$ of $s_{r}$-fold members $C_{s_{r}}\left(s_{r}>1, r=1,2, \ldots, S\right)$. We suppose that these surfaces are themselves irreducible and non-singular; and that, as before, the remaining surfaces of $|C|$ are irreducible.

Denoting, for brevity, a typical multiple surface by $O_{s}$, for each $s$ we have an equivalence $s O_{s} \equiv C$. From the fact that $\left[C^{3}\right]=0$, it follows that $\left[C^{2} C_{s}\right]=\left[C C_{s}^{2}\right]=0$, and also that, for any two surfaces $C_{s}, C_{t}$ (distinct or not), $\left[C_{s}: C_{t}\right]=0$. It may then be shown, as in the analogous theory for surfaces, that, if $P_{g}>0$, each $C_{s}$ is a fixed $(s-1)$-fold component of $K$, and hence that

$$
K \equiv \Sigma(s-1) C_{s}+\left(P_{g}-1\right) C .
$$

It is obvious that, if $S>1$, in general (and certainly when $q_{2}=0$ ) the threefold $V$ must have divisor $\sigma$ greater than unity, since $V$ then contains 
linearly isolated surfaces of which certain multiples are equivalent. When $P_{g}=1, \omega=1$, there may, however, be a pencil of $s$-canonical surfaces, and $V$ then has divisor 1 (Cf. the example in $\$ 4$ ).

By means of the above equivalences for $K$ and for $C_{s}$, we ean at once calculate $P_{i}$ in a case where $P_{g}>0$ evidently we have

$$
P_{i}-1=\Sigma\left[\frac{i(s-1)}{s}\right]+i\left(P_{g}-1\right)
$$

where, as usual, $[x]$ denotes the greatest integer contained in $x$.

Consider now the systems $\left|O^{\prime}\right|,\left|C_{s}^{\prime}\right|$; from the relations $C^{\prime} \equiv C+K$, $C_{s}^{\prime} \equiv C_{s}+K$, we obtain, for $P_{g}>0$,

$$
C^{\prime} \equiv \Sigma(s-1) C_{s}+P_{g} C, \quad C_{s}^{\prime} \equiv \Sigma(t-1) C_{t}+P_{g} C,
$$

the second summation extending only to the $S-1$ surfaces $C_{t}$ other than $C_{s}$.

Thus $C^{\prime}$ and $C_{s}^{\prime}$ are both effective, and cut null curves on $C$ and $C_{s}$ respectively. Hence the latter surfaces have pure canonical curves of order zero; they belong to one of the two elasses described in $\$ 9$, having geometric genera $p, p_{s}$ unity, and arithmetic genera $p, p_{s}$ equal to \pm 1 .

Consider next the case where $P_{g}=0$ if the system $|i K|$, say, is effective, it may be shown, as in the theory of surfaces, that $K$ is given by an equivalence of the form

$$
K \equiv \Sigma(s-1) C_{s}-k C,
$$

where $k$ is some positive integer; the value of $k$ must be determined from special considerations, depending on the particular threefold under discussion. We then have

$$
C^{\prime} \equiv \Sigma(s-1) C_{s}+(1-k) C, \quad C_{s}^{\prime} \equiv \Sigma(t-1) C_{t}+(1-k) C .
$$

Thus each of $C$ and $C_{s}$ is met by the corresponding adjoint in a curve of order zero; and since, by hypothesis, $|i K|$ is effective, $i C^{\prime}$ is likewise effective. If then $p=1, C$ belongs to one of the two classes mentioned above; if, however, $\boldsymbol{p}=0, p=0, C$ must have bigenus $p_{2}=1$, i. e. be an ENRIQUES surface, since if $p_{2}=0, C$ is rational and so cannot possess pluricanonical systems of any index. If, instead, $p=0, p=-1, C$ must belong to the elass of elliptic surfaces, studied by ENRIQUES (4), which possess a pluricanonical curve of order zero; we cannot have $p<-1$, for in that case $C$ is scrollar, and has no pluricanonical carves.

However, if $|i K|$ is effective, we cannot deduce from the above equivalence for $C_{s}^{\prime}$ that $i C_{s}^{\prime}$ is necessarily effective; it may therefore happen that $C_{s}$ has no effective pluricanonical system of any index; in this ease $C_{s}$ is rational or scrollar, according as $p=0$, or $p<0$.

There is one case in which it is possible to state the value of $k$; if $V$ is completely regular, with $P_{g}=0$, then, by $\& 2$, the freedom of the system $\left|C^{\prime}\right|$ 
is precisely $p-1$. Hence, if $p=1$, so that $C^{\prime}$ is effective, we must have $k=1$.

We have next to calculate the invariant $P_{a}$; to this end we observe that, by the formula for the arithmetic genus of a composite surface, we have, for $P_{g}>0$,

$$
\Omega_{2}+1=\Sigma(s-1)\left(p_{s}+1\right)+\left(P_{g}-1\right)(p+1) .
$$

Then, since $2 P_{a}=\Omega_{2}+3$, we obtain

$$
2\left(P_{a}-1\right)=\Sigma(s-1)\left(p_{s}+1\right)+\left(P_{g}-1\right)(p+1) .
$$

Thus, in particular, it follows that

(i) when $p=-1, p_{s}=-1$ (all $s$ ), we always have $P_{a}=1$, whatever may be the value of $P_{g}(>0)$ :

(ii) when $p=1, q_{i}=P_{g}-P_{a}=-\frac{1}{2} \Sigma(s-1)\left(p_{s}+1\right)$, so that

(iii) if, further, $p_{s}=-1$ (all $s$ ), then $P_{g}=P_{a}$.

The principal results which we have just obtained may be summarized as follows:

If the pure canonical system $|K|$, assumed to be effective, is compounded of a rational pencil, without base points, whose only reducible surfaces are a certain number of multiple s-fold members (irreducible and non-singular), then $|K|$ has such surfaces for fixed $(s-1)$-fold components. Each surface of the pencil, whether simple or multiple, has geometric genus unity, and is either regular of genera and plurigenera unity, or irregular hy, erelliptic. If, instead, $P_{g}=0$, but $P_{i}>0$, for some values of $i$, then the generic surface of the pencil may also have geometric genus zero; in that case it is either an Enriques surface, or is an elliptic surface with a pluricanonical curve of order zero. The multiple members of the pencil may be any one of the above types, or else may be rational or scrollar.

12. We have seen that, in the case where the pencil $|C|$ contains multiple members, it is possible for $C$ to have geometric genus zero, although the surface cannot be rational. In such instances $C$ always has base number exceeding unity, which means that there does not exist a number $d$ as defined in $\$ 10$. In order to classify the corresponding threefolds we may, however, consider the system $|A|$ of minimum virtual grade; there may be several distinct systems of this kind. The same method could be applied in $\$ \S 10,11$, when $C$ is of geometric genus unity but not of general type.

We have so far supposed $|R|$ is compounded of a rational pencil; but much of the foregoing investigation would apply equally to the case where $|C|$ is replaced by an irrational pencil $\{C\}$, with or without multiple members $C_{s}$ : for the most part we should merely have to substitute, in the appropriate 
places, signs of algebraic equivalence for those of linear equivalence. It remains to point out the main differences between the two cases.

One obvious modification has to be made in the relation between $I_{g}^{\prime}$ and the number of eurves composing $K$. Thus, if there are $\vee$ variable curves $C$ in $K$, forming a set of speciality index $i$, and if $\{C\}$ has genus $\pi$, then $P_{g}-1=v-\pi+i$.

Again, since $\{C\}$ euts an irrational pencil of curves on the generic prime section of $V$, we must have $q_{2}>0$; more precisely, by a well known property of irregular surfaces, we have $q_{2} \geq \pi$.

In contrast with this result, a surface of class $C$ (\$1) has an irregularity which, save in a few well defined cases, is equal to the genus of the pencil of elliptic curves which the surface contains. It may be that the threefolds of class III, with certain exceptions, possess an analogous property; but in order to establish it, a study of continuous systems of surfaces would have to be undertaken.

Examples of superficially irregular threefolds of the type now considered are easily constructed; they will be given in $\$ 14$.

Finally, it should be noted that $|K|$ may have fixed components, simple or multiple, with appropriate virtual characters; in this case, however, the results obtained are less conclusive.

13. Representation on a multiple cone. - In practice if often proves more expedient to represent threefolds of class III, for which $|K|$ is compounded of a rational pencil, by a quite different process, which in turn leads to another method of classification. The representation we shall now describe (which has previously been used in 17 ) resembles one that will be required again in $\& 15$.

Suppose, either in $\S 9$ or $\S 11$, that $V$ contains a congruence $\Gamma$, of the first order, of curves $\mathscr{B}$ such that the generic $\mathscr{B}$ meets the generic surface $C$ in $m(\geq 1)$ points. Suppose, further, that we know an irreducible linear system $|B|$ of surfaces, of freedom $r \geq 3$, which belong to $\Gamma$, i. $\theta$. are generated by the curves $\mathscr{B}$; evidently the characteristic curve of $|B|$ is composed of a certain number $n(\geq 1)$ of curves $\mathfrak{B}$.

We now refer the system $|B|$ projectively to the system of primes of a space $S_{r+}$, which pass through a fixed point $O$ and, at the same time, refer similarly $|C|$ to the pencil $\Sigma$ of primes through a fixed $S_{r-1}$ of $S_{r+1}$ which does not contain $O$. We make correspond to the generic point $P$ of $V$ the point $Q$ in which the prime of $\Sigma$ corresponding to the surface $C$ through $P$ meets the line common to the system of primes through $O$ which maps the subsystem of $|B|$ for which $P$ is base point. Since all the surfaces of this subsystem contain the curve $\mathcal{B}$ through $P$, while $\mathscr{B}$ meets the generic $C$ in $m$ distinct points, it follows that the locus of $Q$ is a cone $W$, of order $n$ and 
with vertex $O$. This cone is in $(1, m)$ correspondence with $V$, and its $m$-fold generators map the curves $\mathfrak{B}$.

The cone $W$ is of course birationally equivalent to the product of a rational curve and a surface $F$ whose points map the curves of $\Gamma$. Hence, with the notation of $\S 4$, we see that $W$ has geometric genus zero and arithmetic genus $-p_{a}$; also that the superficial irregularity of $W$ is equal to the irregularity $p_{g}-p_{a}$ of $F$.

In this representation of $V$ on $W$ there will in general be a branch surface on $W$, possibly reducible, and consisting of components of various multiplicities. If we suppose that the generic curve $\mathscr{B}$ has genus $g$, then the corresponding generator of $W$ must have $d$ virtual intersections with the branch surface, where $d=2 g-2+2 m$; one or more of these intersections may of course fall at 0 .

The characters required for the classification of $V$ by this method will evidently fall into three distinct groups, as follows.

(1) First, we have the characters of $|C|$, as already described.

(2) Secondly, we have the characters of $\Gamma$; these are the genus $g$ of $\mathfrak{B}$ and the invariants of $F$.

(3) In the third place, there are the characters which depend on both $|C|$ and $\mathrm{r}$, namely

(a) the number $m$, (b) the characters of $|B|$, including the number $n$ and (c) the various numbers required for the specification of the branch surface.

It will be noticed that this representation is analogous to the one employed by ENRIQUEs (4) in his discussion of the irregular surfaces of genus zero, which are all representable on a multiple elliptic cone, The number $m=[\mathscr{B} C]$ corresponds to the determinant of this class of surfaces.

14. Examples. - We shall now illustrate some of the types which have been encountered in the preceding investigation; examples $(i)-(v)$ deal with the product $V=\mathfrak{C} \times F$, for which we use the notation of $\S 4$. In $(i)-(i v)$ $\mathcal{C}$ is elliptic, and, $P_{a}=1$ (C. f. the remarks made in $\$ 6$ ); also the surfaces $C$ and $C_{s}$ are all special J $\mathrm{J}_{\mathrm{ACOBI}}$ surfaces, products of two elliptic curves.

(i) Suppose that $F$ has invariants $p_{g}=p_{a}>1, p^{(1)}=1$, and that the canonical system contains a certain number of $s$-fold elliptic curves. We then have $P_{g}=p_{g}, q_{2}=1$, and $K \equiv \Sigma(s-1) C_{s}+1-\left(p_{g}-1\right) C$, where each $C_{s}$ is the product of $\mathfrak{C}$ with an $s$-fold elliptic curve of $F$.

(ii) If, instead, we take $p_{g}=p_{a}=0, p^{(1)}=1, p_{2}>1$, we obtain an infinite series of types for which $P_{g}=0$, and for which $|i K|$ is effective, being compounded of a linear pencil $|C|$ containing surfaces $C_{s}$; this fact follows from GAETA's work (9) on the regular surfaces of determinant 2. Here we have $K \equiv \Sigma(s-1) C_{s}-C$. If, however, we suppose that $p_{2}=1$, in 
which case $F$ is an ENRIQUEs surface. we have only pluricanonical surfaces of order zero; thus $P_{2 i-1}=0, P_{2 i}=1$ In all these cases $q_{2}=1$.

(iii) Let $F$ be a non-scrollar elliptic surface $\left(p_{g}=0, p_{a}=-1, p_{12}>0\right)$. Here $P_{g}=0$,but $|i K|$ is effective for an infinity of values of $i ; q_{2}=2$. In fact, as ENRIQUes has shown, the pluricanonical systems of $F$ are compounded of a linear pencil, containing multiple elliptic curves; from his results we have $K \equiv \Sigma(s-1) C_{s}-2 C$.

(iv) More generally, we may take $F$ to be irregular with $p_{g}>1, p^{(1)}=1$; the canonical system of $F$ is then compounded of a pencil, of genus $p_{g}-p_{a}$, of elliptic curres, which in general contains multiple members. Hence $|K|$ is compounded of a pencil $\left\{C \mid\right.$, of genus $p_{g}-p_{a}$, containing surfaces $C_{\varepsilon}$. In this case we have $P_{g}=p_{g}, q_{2}=p_{g}-p_{a}+1$.

(v) Now assume instead that $\mathcal{C}$ has genus $\pi>1$, and that $p_{g}=p_{a}=$ $=p_{i}=1 . \mathrm{By} \S 4, K \equiv(2 \pi-2) F_{0}$, so that $|K|$ is compounded of a pencil $\{C\}$, of genus $\pi$, of surfaces birationally equivalent to $F$. We have $P_{g}=\pi$, $P_{a}=2 \pi-1, q_{2}=\pi$. If, however, $F$ is hyperelliptic $\left(p_{g}=p_{i}=1, p_{a}=-1\right)$, we obtain $P_{a}=1, q_{2}=\pi+2$. In neither case does $\{C\}$ contain any multiple members.

(vi) We now give an example of a tridimensionally regular threefold for which $|K|$ is componnded of an elliptic pencil of regular surfaces: in this case $l_{a}^{\prime}=2, q_{2}=1$.

Consider a double $S_{3}$ of which the branch surface consists of four quartics of a general pencil; then $|K|$ is mapped by the surfaces of the pencil, whence $P_{g}=2$. Also, since these surfaces cut the complete canonical series on the base curve $c^{15}$ of the pencil, we have $P_{a}=2$. Now the generic surface $F^{ \pm}$of the pencil has no free intersection with the branch surface; hence $F^{4}$ maps either a pair of regular surfaces of genera unity, or an irreducible surface. But since $\boldsymbol{\Omega}_{2}=1$, in the latter ease we should have two surfaces, each of arithmetic genus 1 , in a $(1,2)$ correspondence free from branch points, which is impossible. Hence $F^{*}$ maps a pair of surfaces, and so $|K|$ is compounded of a pencil $\{C\}$; and since there are four surfaces $F^{4}$ for which the corresponding pairs coincide, it follows that the pencil $C ;$ is elliptic.

Consider next the generic double plane in $S_{3}$; on it we have a base curve consisting of four quartics of a general pencil. Thus the canonical system is mapped by quintics passing simply through the 16 base points of the pencil, so that the invariants of the double plane are $p_{g}=6, p_{a}=5, p^{(1)}=1$; thus $q_{2}=1$.

(vii) In order to illustrate the representation obtained in $\S 13$, we may take the threefold $V$ considered at the end of $\$ 10$, which is mapped on a cubic planar threefold $W^{3}$; in the previons notation, the branch surface is denoted by the symbol $6 D+2(P+1) R$, where $P_{g}=P_{a}=P$. 
We may represent $W^{3}$ on $S_{3}$ by means of cubic serolls $F^{3}\left(l^{2} l_{1} l_{3}\right)$ passing doubly through a line $l$ and simply through two line $l_{1}, l_{2}$ which are incident to $l$ and skew to each other; then the surfaces $R$ and $D$ correspond respectively to planes $F^{i}(l)$ and quadrics $F^{*}\left(l l_{1} l_{z}\right)$.

Now there is a web of quadrics $F^{2}\left(l_{1} l_{2}\right)$ whose characteristic curve is a pair of lines incident to $l_{1}$ and $l_{2}$; taking these to be the system $|B|$ of $\$ 10$, we have, with the notation used there, $n=2, m=4(P+1)$. We may thus map $V$ on a double quadric point- cone $W^{2}$, with vertex $O$; the pencil $|C|$ is mapped on a pencil $\left|C_{1}\right|$ of prime sections of $W^{2}$, having an axis which does not contain $O$ and which meets $W^{2}$ in a line-pair $\lambda_{1}, \lambda_{2}$ with common point $L$, say; the planes $\left(O \lambda_{1}\right),\left(O \lambda_{2}\right)$ correspond respectively to the planes $\left(l l_{1}\right)$, $\left(l l_{2}\right)$, and the net of directrices $D$ are mapped by the system $\left|B_{1}\right|$ of prime sections of $W^{2}$ which contain the line $O L$. Thus the branch surface on $W^{2}$ is represented by the symbol $6 B_{1}+2(P+1) C$ : it is of order $4(P+4)$, has $O L$ for 6-fold line, and has $\lambda_{1}, \lambda_{2}$ for $2(P+1)$ - fold lines.

(viii) In all the preceding examples of multiple surfaces $C_{s}$, these have been irregular hyperelliptic; we shall now illustrate the ease where they are regular. While the general problem of construction involves difficult questions concerning the existence of multiple surfaces of given types, it is possible to give examples of regular threefolds which are analogous to the regular surfaces for which the existence of multiple elliptic curves has been effectively established; these all have determinant 2 (ENRIQUES, 4 ; GAETA, 9).

Suppose that the canonical system of $V$ is compounded of a rational pencil $|C|$, where $C$ is a surface with $p_{g}=p_{a}=p_{i}=1$, which contains a (rational) pencil of elliptic curves, $\mathcal{C}$. These curves generate a rational congruence $\Gamma$ of the first order; we shall consider the case in which the curves $\mathcal{C}$ possess an irreducible non-singular bisecant surface $B$.

Through the generic point $P$ of $V$ there passes a unique curve $C$ meeting $B$ in a pair $Q, Q^{\prime}$ of points, in general distinct; let $P^{\prime}$ be the mate of $P$ in the $g_{2}^{1}$ defined by $Q, Q^{\prime}$. Then the pairs $P, P^{\prime}$ define a rational involution $I_{2}$, which may be used to represeut $V$ on a rational double threefold $W ; C$ is thereby transformed to a rational surface, and $B$ is also transformed to a rational surface, since $C$ meets $B$ pairs of points forming a $g_{2}^{1}$, and so generating a hyperelliptic curve $\mathfrak{B}$ of some genus $\pi \geq 0$. This $g_{2}^{1}$ has $2(\pi+1)$ double points.

We consider now the special case where $B$ is fundamental for some linear system of the form $|B+\mu C|(\mu>0)$; we may then take $W$ to be a rational point-cone with vertex $O$, locus of $\infty^{\prime}$ planes. The peneil in the generio $C$ is mapped by a pencil of lines, centre $O$, in the corresponding plane $\tilde{\omega}$, and the generic line of this pencil must have four free intersections with the branch eurve in $\tilde{\omega}$. If in particular we suppose that the curve $\mathcal{B}$ has genus $\pi=0$, the branch curve must have a node at $O$; it is thus a sextic, in general 
without other singularities. A branch surface of the required type may be obtained by cutting $W$ by a sextic primal having a node at $O$ and containing a number of planes of $W$.

Suppose that $|C|$ contains a double surface $C_{2}$ which is generated by a rational pencil $\left|C_{2}\right|$ of double elliptic curves of $\Gamma$; then each curve $e_{z}$ is unisecant to $B$ and, of the four double points of the involution set up by $I_{2}$ on $\mathcal{C}_{z}$, one lies on $B$, and two are intersections of $\mathcal{C}_{z}$ with the coincidence surface. It follows from the work of ENRIQUES and GAETA on surfaces that $\mathfrak{C}_{2}$ is mapped on $W$ by a point $T$ such that $O T$ forms part of the branch surface, and such that $O T$ is tacnodal tangent at $T$ to the residual portion of the surface. Hence $C_{2}$ is mapped by a line $t$, locus of $T$, in a plane $\tilde{\omega}_{2}$ of $W$ which forms part of the branch surface; $t$ is a tacnodal line on the residual portion of the surface, which is touch $d$ by $\tilde{\omega}_{2}$ along $t$. The sextic branch curve in $\tilde{\omega}_{2}$ consists of $t$, connted four times, and a repeated line through $O$, limit of the nodal tangents in the general case.

By the results of $\$ 11$, the geometric genus of $C_{2}$ must be 1 in the case where $P_{g}>0$; and since it contains a rational pencil of elliptic curves, its arithmetic genus must also be 1 . The possibility of constructing examples of threefolds containing surfaces $C_{2}$ depends on that of obtaining branch surfaces with the requisite kind of tacnodal plane; and these certainly exist for surfaces of sufficiently high order, as one can see by representing $W$ on $S_{3}$ in the usual manner.

(ix) An interesting illustration of the representation given in $\S 13$ has for its point of departure the following result, due to ENRTQUES and SEvERI (5). Let $f(x, y, z)=0$ be the equation of a KuMMer quartic surface $F$ in $S_{3}$, and let $\psi(x, y, z)=0$ be the equation of one of the associated tetrahedra, whose vertices are nodes of $F$, and whose faces each contain six nodes in all. Then it may be shown that the surface in $S_{4}$ whose equations, in nonhomogeneous coordinates, are $u^{2}=\psi(x, y, z), f(x, y, z)=0$, is a general $\mathrm{J}_{\mathrm{ACOBI}}$ surface; in other words, we have a representation of the latter on a double KUMMER surface, the branch curve consisting of the neighbourhoods of the 16 nodes.

Consider now the threefold $V$ of $S_{5}$ whose equations, in non-homogeneous coordinates $(x, y z, t, u)$ are

$$
u^{2}=\prod_{1}^{S}\left(t-a_{s}\right) \uplus(x, y, z), \quad f(x, y, z)=0,
$$

where the $S$ numbers $a_{s}$ denote arbitrary constants. The equation $f=0$ may be regarded as that of a cone (cylinder) of $S_{4}$ projecting the surface $F$, and $V$ is represented on this double cone, the branch surface consisting of the surfaces $C_{s}$ eorresponaing to the sections of the cone $f$ by the primes $t=a_{s}$; evidently these are regular surfaces of genera nuity. The generic surface $C$, corresponding to the section $t=c$, say, is, by the above result, a $J_{A C O B I}$ surface. 
To obtain a virtual canonical surface $K$ on $V$ we observe that $f$ may be regarded as the product of $F$ with a rational curve $\mathfrak{C}$, and hence that, by \$ 4. a virtual canonical surface on $f$ is given by $-2 F_{n}$. Thus the surface $K$, which is found by adding the transform of $-2 F_{0}$ to the coincidence surface, satisfies the equivalence $K \equiv \Sigma C_{s}-2 C$. From this, and the fact that $2 C_{s} \equiv C$, $P_{i}$ may be calculated in the usual manner; in particular, we have $P_{g}=0$. The formula for $P_{a}$ gives, on substituting $p=-1, p_{s}=1, P_{a}-1=S$.

The congruence $\Gamma$ of eurves $\mathscr{B}$ is mapped on the double generators of $f$, which are given by $x=a, y=b, z=c, f(a, b, c)=0$; the curves themselves are thus given by the equation $u^{2}=\Pi\left(t-a_{s}\right) \psi(a, b, c)$.

15. Threefolds of class IV. - Suppose that $V$ possesses an effective pure canonical system $|K|$ which, by reason of the relation $\omega=4 \Omega_{0}+1$, we may assume to be effectively free from base points; and suppose also that the characteristic curve of the system, which has freedom $P_{g}-1 \geq 2$, consists of $k(\geq 1)$ elliptic curves $\mathscr{K}$, forming a congruence $\Gamma$ of the first order. On the generic $K, \mid K$ cuts a pencil, rational or irrational, of curves $\mathcal{K}$; by virtue of the equivalence $K^{\prime} \equiv 2 K,|K|$ cuts a semicanonical system on $K$, the canonical system itself being componnded of $2 k$ eurves of the pencil: since, by hypothesis, $|K|$ is without base points, this canonical system has no fixed components.

It follows from $\S 1$ that the surface $K$ is regular if, and only if, the pencil of curves $\mathfrak{A}$ contained in it is rational; in this case $\Omega_{2}=2 k+1$, and, since $2 P_{a}=\Omega_{2}+3$, we have $P_{a}=k+2$. If, instead, the pencil $\{\mathfrak{A}\}$ is irrational and of genus $g$, it again follows from $\S 1$ that the geometric genus $p$ of $K$ is given by $p \geq g+Q_{2}$; moreover, assuming that the sets of $2 k$ curves which form the canonical system of $|K|$ have speciality index $j$ in $\mid \mathfrak{T}\}$, we deduce from the RIEMANN-Roch theorem that $p-1=2 k-g+j$.

Consider the linear system, of freedom $P_{g}-2$, ent by $|K|$ on the generic surface $K$; we have, by the RiEmann-Roch theorem,

$$
P_{g}-2=k-g+i-\delta,
$$

where $i$ denotes the speciality index in $\mid \mathfrak{d}$ ! of the sets of $k$ curves which form the characteristic curves of $|K|$, and $\delta$ is the deficiency of the characteristic system: this satisfies the inequality $\delta \leq q_{2}$ (B. SEgre, 19!.

We now observe that

If $K$ is regular and $q_{2}=0$, then $P_{g}=P_{a}$.

For, if $q_{3}=0$, the characteristic system of $|K|$ must be complete; and, if $K$ is regular, $g=0$, whence $P_{g}-2=k=P_{a}-2$. Hence $V$ is completely regular.

It should, however, be noted that $V$ may be completely regular even when $K$ is irregular. Consider, for example, the double $S_{3}$ with a branch 
surface $F^{2 n}\left(O^{2 n-4}\right)$ having a $(2 n-4)$-fold point $O$, but no other singularity. In this case $|K|$ is mapped by cones $F^{n-4}\left(O^{n-4}\right)$; and, for $n \geq 7$, each such cone contains an irrational pencil of lines representing elliptic curves on $V$ which belong to a rational congruence of which the characteristic system of $|K|$ is compounded. We have $k=(n-4)^{2}, g=\frac{1}{2}(n-5)(n-6)$, $P_{g}=P_{a}=\frac{1}{2}(n-2)(n-3), q_{2}=0$, this last result being a consequence of the fact that the generic double plane in $S_{3}$ is regular.

We proceed to calculate the plurigenera $P_{m}$ of $V$ on the assumption that $q_{2}=0$; in that case $K$ will in general be irregular, but we know that the characteristic systems of all the complete systems $|m K|(m \geq 1)$ are complete (B. SEGRE, 19). Since the former systems are all compounded of $\Gamma$, it follows that each system $|m K|$ must eut a complete system (compounded of the pencil $\{\mathfrak{K} \mid)$, on the generic $K$. If, then, we denote the freedom of this system by $r_{m}$, we have

$$
P_{m}-1=r_{m}+P_{m-1} \quad\left(m=2,3, \ldots ; P_{1}=P_{g}\right) \text {. }
$$

Suppose that the sets of $m k$ curves which form the system cut on $K$ by $|m K|$ have speciality index $i_{m}$ in $|\mathfrak{H}|$; then $r_{m}=m k-g+i_{m}$, and thus

$$
P_{m}-1=m k-g+i_{m}+P_{m-1} \text {. }
$$

From this relation $P_{2}, P_{3}, \ldots$ can be found in succession. All that can in general be asserted concerning the numbers $i_{m}$ is that $i_{m}=0$, for all $m>m_{0}$; if however, $g=0$, or $g=1$, then $i_{m}=0$ (all $m$ ).

In all that has preceded we have supposed that the system $|K|$ has freedom 2 at least; but there exist threefolds for which $P_{i} \leq 1(i=1,2, \ldots, j-1)$, $P_{j} \geq 3$, such that the characteristic system of $|j K|$ is compounded of a con. gruence of elliptic curves. (The case where $P_{i}=2$, for some value of $i$, merely leads to class III). We may then apply the above considerations to the system $|j K|$.

Examples of such threefolds will be given later. The question naturally arises: can $j$ be arbitrarily large? The answer to the analogous question for surfaces is that it cannot be. Thus, if a surface has genus $p_{g}=1$, with a canonical curve of order greater than zero, we must have $p_{\mathrm{z}} \geq 2$ : if instead $p_{g}=p_{a}=0$, and there is a bicanonical curve of order zero, we do not obtain a surface of the requisite type: if, however, there is a bicanonical curve of order greater than zero, $p_{s} \geq 2$. Again, in the case where $p_{g}=0, p_{a}=-1$, any pluricanonical systems of order greater than zero must be compounded of a rational pencil of elliptic curves; and in all such cases we have $p_{12} \geq 2$ (ENRIQUES, 4).

16. The elassification of $V$. - We have seen that the surface $K$ belongs to the class $C$ defined in $\S 1$, and therefore that there is attached to it a certain determinant $d \geq 1$. As we have remarked, the general surface of this 
class depends, not only on $d$, but also on the nature and number of the multiple elliptic curves contained in the canonical system $|\mathscr{H}|$. In the present case, however, such curves cannot exist; for an $s$-fold curve $\mathfrak{H}_{s}$ on the generic surface $K$ would have to be an $(s-1)$-fold component of $|\mathcal{H}|$; hence by virtue of the equivalence $K^{\prime} \equiv 2 K$, it must be a fixed component of the system cut by $|2 K|$ on $K$. and hence also of the characteristic system of $|K|$ : whereas $|K|$ is effectively free from base points. Even if we allowed $|K|$ to acquire $\mathfrak{K}_{s}$ as an unassigned $s$-fold base curve, it would be impossible to secure the passage of $|2 K|$ through $\mathfrak{R}_{s}$ with the required multiplicity. We can obtain such multiple ourves only by allowing $|K|$ to have fixed multiple components (7).

In the case $P_{g}>3$, we may obtain a representation of $V$ on a multiple cone by substantially the method employed in $\S 13$ : choosing a pencil $\mid A$ of irreducible non-singular surfaces which are m-secant to the curves of $\Gamma$, we identify this with the pencil $|O|$ of $\S 13$, and at the same time identify $|K|$ with the system $|B|$. We thas map $V$ on an $m$-fold cone $W$, of order $k$ and with vertex $O$, which is normal in $\left[P_{g}\right]$, and the curves $\mathfrak{H}$ are mapped on the $m$-fold generators of $W$. The latter meet the branch surface in $2 m$ free points.

The same method may be used in the case $P_{g}=3$, provided that $k=1$; we then have a representation on an $m$-fold $S_{3}$

For the purposes of elassifieation we require, to begin with, in addition to the characters already introduced, the minimum possible value of $m$. It is slear that, in all cases, $m \geq d$, and that, when $d=1, m \geq 2$; for if, when $d=1$, there were a rational pencil of curves unisecant to $\mathcal{H}$, then $\mathfrak{H}$ would be rational.

Assuming that $K$ is a general surface of determinant $d$, it follows from GAETA's work (9) that, on $K$, a base is provided by a curve $\mathcal{K}$ and a $d$-secant $\mathscr{D}$ of minimum virtual grade; the characters $[\mathfrak{D}]=\pi,\left[\mathfrak{D}^{*}\right]=\nu$, of the system $|\mathfrak{D}|$ are absolute invariants of $K$.

Using these results, we can obtain some preliminary information concerning the pencil $|A|$. Denoting by $|\mathfrak{A}|$ the pencil of curves in which $|A|$ meets the generic surface $K$, we must have an equivalence (linear or algebraic) of the form $\mathfrak{A} \equiv \lambda \mathfrak{D}+\mu \mathfrak{K}$, where $\lambda$ and $\mu$ are integers. Since, by hypothesis,

$$
[\mathfrak{D O H}]=d, \quad[\mathfrak{E} \mathfrak{J}]=m, \quad\left[\mathfrak{K}^{*}\right]=0, \quad \text { on } \quad K,
$$

we have $[\mathfrak{A O K}]=\lambda[\mathfrak{D} \mathfrak{d}]$, or $m=\lambda d$. Thus $\lambda$ and $d$ must both be divisors of $m$; and $\lambda=m / d$.

With the notation of $\S 10$ for the characters of the system $|A|$, and writing $A K=[\mathfrak{Q}]=\rho,\left[A^{2} K\right]=\left[\mathfrak{Q}^{2}\right]$, we obtain

$$
\rho=[\lambda \mathscr{D}+\mu \mathfrak{O} \mathrm{l}]=\lambda(\pi-1)+\frac{1}{2} \lambda(\lambda-1) \nu+\lambda \mu d+1, \quad\left[\mathfrak{Q}^{2}\right]=\lambda^{2} \nu+2 \lambda \mu d .
$$

(7) As, for instance, in the case of the elliptic threefolds, which are studied in a forthcoming paper. 
Hence, if $\rho$ is known, $\mu$ and $\left[\mathfrak{Q}^{*}\right]$ are uniquely determined.

As in $\$ 10$, we deduce from the equivalence $A^{\prime} \equiv A+K$ that

$$
\begin{aligned}
& p^{(1)}=\left[A(A+K)=\pi+\rho+\left[\mathfrak{Q}^{2}\right]-1,\right. \\
& p^{(1)}-1=\left[A(A+K)^{2}\right]=n+2\left[\mathfrak{Q}^{3}\right]+k m .
\end{aligned}
$$

In the case where $K$ is regular, we have the following simplifications:

(i) From the equivalence, on $K, \mathscr{D}^{\prime} \equiv \mathscr{D}+\left(\Omega_{z}-1\right) \mathfrak{d}$, we obtain

$$
\left[\mathfrak{D}^{\prime} \mathfrak{D}\right]=2 \pi-2=v+\left(\Omega_{2}-1\right) d \text {. }
$$

This provides a relation between the characters $\pi$ and $v$.

(ii) Since the pencil of curves $\mathfrak{H}$ on $K$ is rational, $W$ must have rational curve sections; hence $W$ is either plane-ruled or, if $k=4$, projects a VERONESE surface from an external point. In either case $|K|$ contains subsystems which can be utilized in the discussion (Cf. 17).

(iii) If, further, $V$ is completely regular, $W$ must be normal; we can then with advantage introduce the character $r$, representing the freedom of the complete system $|A|$, and hence, as in $\S 10$, obtain an inequality for $p$.

Evidently the same considerations could be applied to the examination of the system $|j K|$, in a case where $P_{g} \leq 3, P_{j}>3$.

17. Examples - The various points that have arisen in the previous discussion are illustrated by the following examples.

(i) A priori, the simplest case that can arise is that in which $g=0$, $d=1$. Actually, this cannot occur; for we should then have, on the generie $K$, a unique unisecant curve $\mathcal{H}$ to the pencil $|\mathfrak{H}|$. (There could not be a pencil, rational or irrational, of such curves). If $K$ varied in a pencil, the locus of $\mathcal{Q} \mathfrak{l}$ would be a rational surface $U$; hence, since $|K|$ has freedom 2 at least, we should be able to construct a rational pencil $|U|$ of rational surfaces, in contradiction to the hypothesis $P_{g}>0$.

In the case $g>0, d=1,2 \ell$ would have genus $g$, and the above reasoning would not apply.

(ii) Suppose now that $g=0, d=2$, and that $q_{\mathrm{g}}=0$ (whence $q_{1}=0$, also). An example in which $m=2, P_{g}=P_{a}=P=3$, is afforded by the double $S_{3}$ with branch surface $F^{10}$ having an ordinary 6 -fold point $O$; in this case $k=1, \Omega_{2}=3$. The system $|A|$ mapped by the double planes of $S_{3}$ is composed of regular surfaces, having characters $p=6, p^{(1)}=9, n=2, \pi=4$. The system is therefore regular.

If $P>3$, the other characters of $V$ remaining as before, we shall have a representation on a double rational normal cone $W^{P-2}$ of $[P]$, in which $|K|$ is mapped by the double sections through the vertex $O$. If, as we shall assume, $W$ is plane-ruled, the branch surface must meet the generating planes in curves of order 8 at least; for if, for instance, the curves were sextics, we should have either a threefold of class III or some further simplification. 
We can construct a one-parameter family of threefolds of class IV, corresponding to the case where the branch surface is the intersection of $W^{P-2}$, residual to $2(P-4)$ generating planes, with an octavic primal $\Phi^{8}$ which has a 4-fold point at the vertex $O$.

To show that this double threefold has the required character, we map the generic section $R^{P-2}$ of $W^{P-2}$ through $O$ by means of the system $e^{P-2}\left(O_{i}{ }^{P-3} O_{i}\right)$ of plane curves, where $O_{i}(i=2,3, \ldots, P-2)$ are $P-3$ points consecutive to $O_{1}$. Then the section of $R^{P-z}$ by $\Phi^{8}$, residual to $2(P-4)$ generators, is mapped by a eurve $e^{6 P-8}\left(O_{i}^{6 P-16} O_{i}^{8}\right)$; if $\Phi^{8}$ now acquires a 4 -fold point at $O$, this eurve becomes $\mathcal{C}^{6}{ }^{6-8}\left(O_{1}^{6 P-12} O_{i}^{4}\right)$. Now a double plane with such a branch curve maps a regular surface of linear genus $\omega=1$, and arithmetic genus $\mathrm{Q}_{2}=2 P-3$; for the canonical system is represented by the curves $\mathcal{C}^{3 P-7}\left(O_{1}{ }^{3 P-7} O_{i}\right)$, and these break up into $P-3$ lines $O_{1} O_{i}$, together with the curves $\mathcal{C}^{2 P-4}\left(O_{i}{ }^{2 P-4}\right)$.

A surface $A$ is mapped on a donble (non-conical) scroll $R^{P-2}$; the branch curve is cut by $\Phi^{8}$, residual to $2(P-4)$ generators, so that $p=3 P-3$, $p^{(1)}-1=8(P-2)$, and $n=2(P-2), \pi=3$.

(iii) Another family of threefolds can be constructed by taking a double $S_{3}$ whose branch surface $F^{2 \nu}\left(O^{2 y-4} ; l^{2 y-8}\right)(v \geq 7)$ has an ordinary $(2 v-4)$-fold point $O$, and an ordinary $(2 v-8)$-fold line $l$ through $O$. The canonical system $|K|$ is mapped by the rational cones $F^{v-4}\left(O^{\nu-4} ; l^{\nu-5}\right)$, whence $k=2$, $p=2 v-7, \Omega_{2}=4 v-17$. The system $|A|$ mapped by the double planes of $S_{3}$ has characters $p=3(v-3), p^{(1)}=8(v-4)-1, n=2, \pi=v-1$.

(iv) As an example of the case $q_{2}=0, q_{1}>0$, consider the duable $S_{3}$ with branch surface $F^{12}$ which has ordinary 6 -fold points at 8 associated points common to a net $|Q|$ of quadrics. Then $|K|$ is mapped by $|Q|$, from which it follows that $P_{g}=3, P_{a}=2, q_{2}=0$. Denoting any three linearly independent quadrics of $|Q|$ by $Q_{1}, Q_{2}, Q_{3}$, we may write the equation of $V$ in the form $t^{2}=f_{6}\left(Q_{1}, Q_{2}, Q_{3}\right)$, where $f_{6}$ is a sextic form with constant coefficients. We thus see that the generic characteristic curve of $|Q|$ maps a pair of elliptic curves $\mathfrak{H}$, so that $k=2$; and that, in the pencil of characteristic curves on a fixed surface $K$, there are 6 coincident pairs : hence the pencil $\{\mathfrak{d}\}$ on $K$ has genus $g=2$. Since $P_{a}=2$, we have $\Omega_{2}=1$, whence $p=3$. These results agree with the formulae of $\S 15$; for, with the previous notation, $i=1, \delta=0$.

(v) A wide range of types is illustrated by the product $V$ of an elliptic curve $\mathcal{C}$ and a surface $F$ (possibly irregular), with a canonical system which is free from base points, and which is not compounded of a pencil. With the notation of $\S 4, P_{g}=p_{g}, P_{a}=1, q_{2}=p_{g}-p_{a}+1, \Omega_{2}=-1$; also $p=p^{(1)}$, and $g=p^{(1)}$.

Among these threefolds there are examples of base number 2, which are analogous to scrollar surfaces of general type; for instan.e, we may suppose $F^{r}$ 
to be a general surface of order $m(>3)$ in $S_{3}$, in which case its base is a single carve $\mathfrak{F}$; then $V$ has a base consisting of $F_{0}$ and $\mathcal{C} \times F$.

(vi) By a similar method we can construct examples of threefolds of class IV for which $P_{g} \leq 1$. With the notation of $(v)$, consider the threefold $V$ for which $F$ is the surface, discovered by CAMPEDELLI (ENRIQUES, 4), with invariants $p_{g}=p_{a}=0, p^{(1)}=p_{2}=3$; this is mapped on a double plane with a branch curve (possibly reducible) of order 10 , endowed with $6(3,3)$ points: a branch curve of this kind can be constructed by taking three conics two by two bitangent, and a quartic which is tangent to each conic at each point of contact. There is a net of bicanonical curves on $F$, corresponding to the quartics through the 12 triple points of the branch curve.

It follows that $V$ has invariants $P_{g}=0, P_{a}=1, q_{1}=1$; and that the system $|2 K|$ is a net whose characteristic system is compounded of a congruence of elliptic curves; a surface $2 K$ is the product of an elliptic curve and a curve of genus 3 . We have $P_{2}=3, k=p^{(1)}-1=2$.

Next, consider the case where $F$ has a single canonical curve of order greater than zero, and at least a net of bicanonical curves, not compounded of a pencil; here $P_{g}=1, P_{2}=p_{2}$, and $k=p^{(1)}-1$.

However, it is a consequence of the known properties of surfaces ( $\$ 15)$ that, if there exist threefolds of class IV for which $P_{3} \leqslant 1$, they cannot be constructed by the present method.

\section{BIBLIOGRAPHY}

1. L. Campedelut, «Rend. Sem. Mat. Padova ", (6), (1985), 57.

2. G. Castellnuovo, Rend. Acc. Lincei », (5), 6, (1897), 372; 406; Memorie scelte, (Bologna. 1937), p. 443.

3. F. Conforto-F. Gherardelli, - Annali di Mat. ", (4), 33, (1952), 273.

4. F. ENRIQUes, Le superficie algebriche, (Bologna, 1949).

5. F. Enriques-F. Severi, "Acta Math.", 32, (1909), 283.

6. G. FANo, "Mem. Ace. Italia ", 8, (1937), 23.

7. - - Comm. Pont. Ac.", 11, (1947), 635.

8. - - «tti Congr. Internaz", (Bologna, 1928), IV, (1981), 115.

9. F. Gaeta, "Mem. Acc. XL", (4), 2, (1951), 1.

10. - - Annali di Mat. ", (4). 33, (1952), 91.

11. S. Lefschetz, "Trans. Amer. Math. Soc. „, 22, (1921), 327.

12. B. D'ORGEVAL, «Journ. de Math. s, (9), 25, (1946), 173.

13. L. Roth, "Rend. di Mat. ", (5), 10, (1951), 297.

14. - - Rend. Acc. Linceì », (8), 12, (1952), 265.

15. - - Rend. Acc. Lincei ", (8), 12, (1952), 401.

16. - - Rend. Acc. Lincei ", (8), 9, (1950), 246.

17. - - Rend. Acc. Lincei *, (8), 12, (1952), 66.

18. B. Segre, "Mem. Acc. Italia", 5, (1934), 479.

19. - " Mem. Ac. Belgique», (2), 14, (1986), 1.

20. F. Severi, "Rend. Palermo », 28, (1909), 33.

21. - - Annali di Mat.", (4), 32, (1951), 1.

22. - - Funzioni quasi-abeliane, (Roma, 1947). 